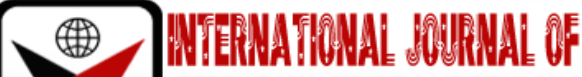

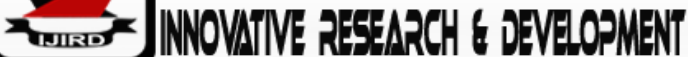

ISSN 2278-0211 (Online)

\section{Assessment of the Physical, Chemical and Biological Properties of Sachet Water in Mubi Metropolis, Adamawa State North East, Nigeria}

\author{
Kadmiel Oliver \\ Lecturer, Department of Geography, Federal College of Education Yola, Nigeria \\ Micheal William Malawi \\ Lecturer, Department of Geography, Nigeria Army University Biu, Nigeria \\ Hajjatu Tammi \\ Lecturer, Department of Geography, Adamawa State University Mubi, Nigeria
}

\begin{abstract}
:
Drinking sachet water quality was investigated in Mubi metropolis, Adamawa state, Nigeria, to ensure the continuous supply of clean and safe drinking water for the public health protection. For that reason, detailed biological, physical and chemical analyses of drinking water samples were carried out in different companies that produce sachet water in Mubi metropolis, Adamawa state. A number of parameters such as $\mathrm{pH}$, turbidity, conductivity, total suspended solids (TSS), total dissolved solids (TDS), and heavy metals such as $\mathrm{Cu}, \mathrm{Zn}, \mathrm{Mg}, \mathrm{Fe}, \mathrm{Cd}, \mathrm{Pb}, \mathrm{Cr}, \mathrm{As}, \mathrm{Hg}$, and Sn were analyzed for each water sample collected period the period of five weeks. The obtained values of each parameter were compared with the standard values set by the World Health Organization (WHO) and local standards such as National Drinking Water Quality Standard (NDWQS). The values of each parameter were found to be within the safe limits set by the WHO and NDWQS. Overall, the water from all the locations was found to be unsafe as drinking water. NAFDAC should carry out regular and frequent surveys of sachet water quality analysis for proper monitoring and management of sachet water production and its quality, Sachet water producing companies should endeavor to carry out proper treatment of the water they produce to ensure it is free of pathogens. Due to the associated effect of excess nitrate in drinking water on infants, it is recommended that some of these sachet waters be avoided for infant's consumption. This study recommends further studies to investigate the life of sachet water in the area beyond 5 weeks and to critically examine the biological quality of the sachet water produced in the area.
\end{abstract}

Keywords: Biological parameters, chemical parameters physical parameters, and Water quality

\section{Introduction}

Water is an indispensable resource that is needed for the continued existence of all living things including man. Human life depends to a large extent, on water. It is used for an array of activities such as domestic, industrial, agricultural, transportation and others. Adequate supply of fresh and clean drinking water is a basic need for all human beings (Edema, et al., 2011). One of the major and critical problems in most developing countries today is the provision of inadequate and safe drinking water to its populace (Kulshershtha, 1998). Most people living in the major cities of Nigeria do not have access to pipe borne water, either it is unavailable or inadequate where obtainable (Omalu, et al., 2010). Therefore, people resorting to the costlier alternative of buying water from vendors, sachet or bottled water has become a major source of drinking water. Sachet water, a brand of packaged water, has gradually become the most widely consumed source of drinking water for both the rich and the poor in Mubi metropolis, Adamawa state and Nigeria at large.

It is the brand of choice to most people because it is cheaper alternatively to the bottled brand. Hygiene, purity, tastes and most importantly, safety is probably amongst various reasons for sachet water consumption. Unfortunately, the problems of its purity and health concerns have begun to manifest (Oladipo,et al., 2009). That is why assessment of the physical, chemical and biological properties of sachet water should be inevitable and non-temporal. Water pH is simply the alkalinity or acidity of water and plays a major role on water suitability and usage (World Health Organization, 2003, Adline, and Alaso, 2015).Electrical conductivity (EC) is a measure of ability of water to conduct electricity (Pankaj, Nihar, Mihir, and Malabika, 2015). According to Isikwu, and Chikezie (2014), the EC value of water depends on the concentration and degree of dissociation of electrolytes and it gives a good idea of the amount of dissolved material in the water. Turbidity is determined by suspended solid particles such as clay, silt, organic and inorganic matter, soluble coloured organic compounds, plankton and microscopic organisms in water (Minnesota Pollution Control Agency, 2008). According to Isikwue and Chikezie (2014), the total hardness is an indication of the presence of calcium, magnesium salts or both in water. 
Total dissolved solid (TDS) is the term used to describe the inorganic salt and small amount of organic matter present in water (Mohammad, Mohammad, Tanzina, Shafkat, Rabiul, Barun, 2016). The principal constituents are usually calcium, magnesium, sodium and potassium cation, carbonate, hydrogen carbonate, chloride, sulphate and nitrate anion (WHO, 1996; Mustafa, et al., 2013).Dissolved solids in water can affect the suitability of water for drinking, domestic, industrial and agricultural uses (Rahmanian, Siti, Homayoonfard, Ali, Rehan, Sadef, and Nizami 2015, Patterson, Johnson, 2003). At higher levels, drinking water may have unpleasant taste, odour, and cause gastrointestinal distress and have laxative effects on consumers (Oteze, 1991; WHO, 1996; Isikwue, and Chikezie, 2014). However, consumption of water with extremely low TDS level is not recommended as it has been reported to exhibit flat insipid taste (Bruvold, and Ongerth, 1969; WHO, 1996) and may also lead to some health challenges such as goiter, hypertension, ischemic, heart disease among others, especially, in the presence of poor dietary habits (Akpoborie, and Ehwarimo, 2012).According to Isikwu, and Chikezie (2014), chlorine is invariably present in small amounts in almost all water body and its contents go up appreciably with increasing salinity. However, excessively high concentration of chlorides is an indication of pollution (Nayla, 2019). Lack of information on physical, chemical factors and biological organisms associated with drinking water creates some uncertainties in our understanding of the overall quality of drinking water in our markets. In essence, although sachet water is readily available and affordable, there are concerns about its purity. This issue is of public health significance due to the continuous increase in the sale and indiscriminate consumption of sachet unwholesome drinking water in Mubi metropolis, Adamawa state and Nigeria at large. This prompted the researcher to assess thephysical, chemical and biological properties of sachet water in Mubi metropolis, Adamawa state of Nigeria.

\subsection{Study Area}

The study was conducted in Mubi metropolis, Adamawa state, Nigeria. The area is located between latitude $9^{\circ} 30^{\prime}$ and $11^{\circ}$ north of the Equator and longitude $13^{\circ} 20^{\prime}$ and $13^{\circ} 35^{\prime}$ East of Greenwich Meridian (figure 1). Mubi metropolis, Adamawa state accommodates the dry land areas of Mubinorth and south, Adamawa state. Mubi metropolis, Adamawa state is bounded to the south by Maiha local government, to the east by Cameroun Republic, to the north by Michika local government and to the west by Hong Local Governments Area. The area is located at an elevation of 1200 to 1500 meters above sea level(Yonnana, Adebayo, and Mahmud, 2008).

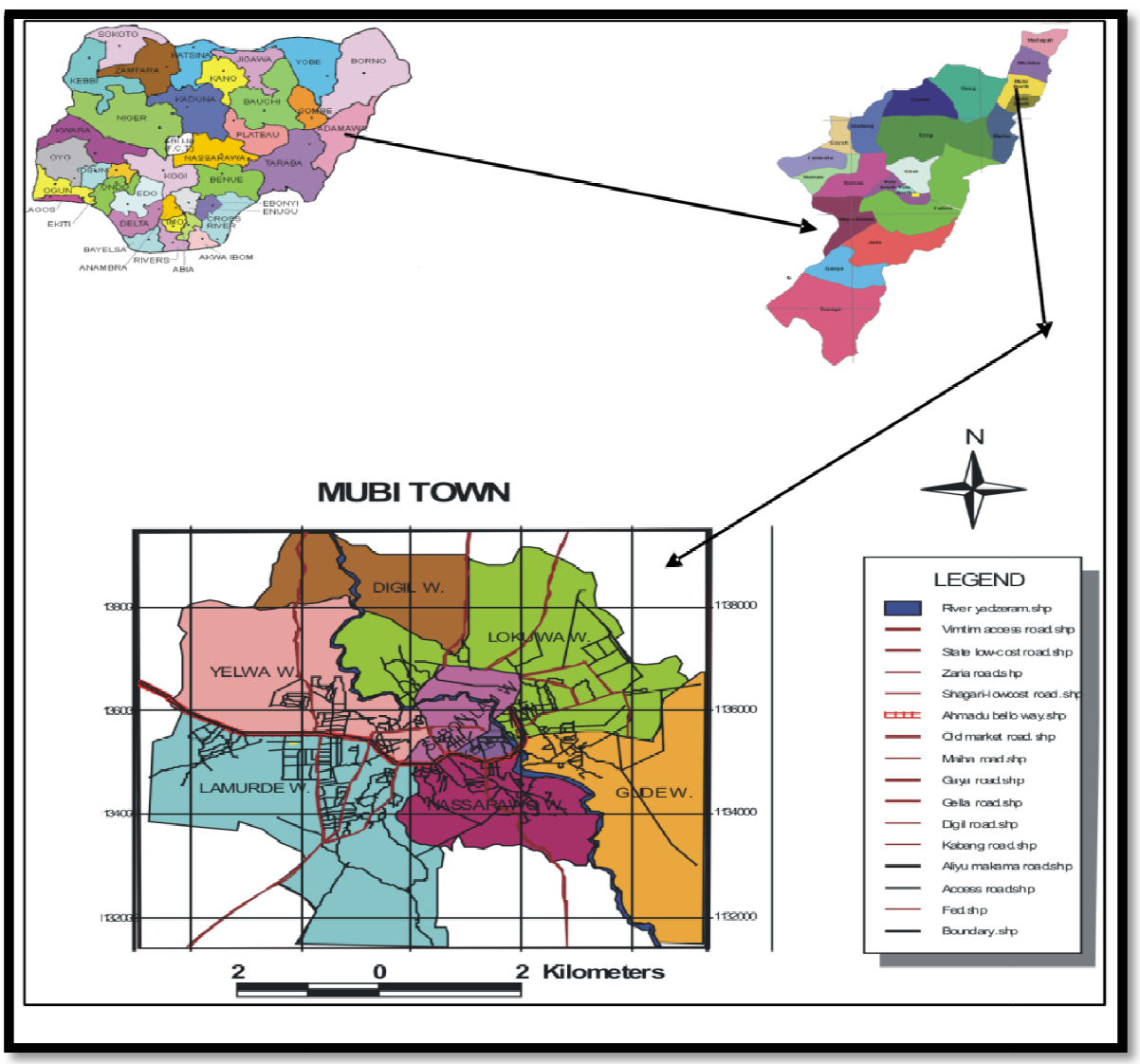

Figure 1: Mubi Metropolis, Adamawa State

Source: Researcher Extracts (2021)

The land surface is generally undulating. According to Adebayo (2014), many rivers, including the Yedzeram River from which the state derives its water sources for drinking, have their source in the eastern part of the metropolis. Muvir and Njairi Rivers are also tributaries to the Yedzeram River flows across the Mubi town. According to Yonanna, et al, (2020), the climate of the area is of the tropical savanna type with distinct wet and dry seasons. Rainfall pattern of the area is generally characterized by long and short rainy season with short and long dry season. The long rainy season starts in May and lasts to the end of October, with a peak period in August. The mean annual rainfall for the region is about 1200 $\mathrm{mm}$. The mean monthly temperature is about $37^{\circ} \mathrm{C}$ with a mean monthly range of $8^{\circ} \mathrm{C}$. The mean relative humidity is over 
85\%. The study area is exclusively located in the Basement Complex of the northeastern Nigeria. The basement was last affected by the $600 \pm 150$ Ma Pan-African orogeny which resulted into plates' collision between the passive continental margin of the West African craton and the active Pharusian continental margin (Dada 2006). According to Adediji and Ajibade (2005), the study area is underlined by six rock types. These rocks types include pegmatite (P) and Schist, Pegmatised (Sp), Gneiss and Migmatite, undifferentiated (M) and Porhyroblastic gneiss (OPg), Charnockitic metal intrusive (CL/M) and Quartzite and quartz-schist (Q).

The vegetation of the area is in the tropical Savanna belt region. It is characterized by tufted savannah grasses and dotted with trees especially mahogany (Symth and Mongomery, 1962). Although, much of the natural vegetation has been depleted largely due to crop cultivation, which is the dominant economic activity in the area. Hunting is an important traditional occupation in Mubi metropolis, Adamawa state, and this has led to considerably dwindling of the game population of the area (Adewale, 2017). The notable species found in the area include mouse, rat and squirrel. Yonanna,et al, (2020) reported that the soils of the area belong to the highly ferruginous tropical red soils associated with basement complex rocks. As a result of the dry humid savanna cover in the area, the soils are generally deep and of two types, namely, deep clayey soils formed on low smooth hill crests and upper slopes; and the sandy hill wash soils on the lower slopes. The well drained clay soils of the hill crest and slopes are very important, because they provide the best soils for cocoa cultivation in the area. The lighter loams are more suitable for cultivating the local food crops such as yam, cassava and maize. Soil degradation and soil erosion are generally not serious in the area, but considerable hill wash is recorded along the slopes of the hills.

\subsection{Materials and Methods}

DR/2000 instrument,thermometer,Digital pH meter,Hatch conductivity meter 4600,potable turbidity meter, standard EDTA, incubation, spectrophotometer, potassium chromate,alcohol, test strip kit, pour plate, membrane, Eosin.

Seventeen sachet water factories are presently registered with NAFDAC in Mubi metropolis, Adamawa state. Using sample size calculator to calculate the number of factories to be sampled, seven pure water factories was used as the sample size for this study. Thus, seven water brands with NAFDAC certification were randomly selected for this study. Two bags of each brand of sachet water, containing twenty sachets each, was procured from each factory immediately after production, it was labeled and transferred to the laboratory for standard analysis. These samples were labeled SW1 SW7. The samples were stored at room temperature. The physical, chemical and microbial quality of the water samples was examined at intervals of two weeks after the week of production to a period of fifth weekto examine if the quality of the water deteriorates with time.

\section{Methods of Data Analysis}

\subsection{Laboratory Analysis}

Laboratory analysis was carried out on water samples to provide relevant information necessary to achieve the aim and objectives of this study. Essential water parameters such as colour, pH, hardness, total alkalinity, dissolved oxygen, organic constituents among others that determines the quality of water (Denloye, 2004) was analyzed on the week of production, third week and fifth week. Laboratory analysis was carried out using standard procedure as shown in Table 1.

\begin{tabular}{|c|c|c|}
\hline $\mathbf{S} / \mathbf{N}$ & Test & Method and Procedures \\
\hline 1. & Colour & $\begin{array}{l}\text { This was measured using the Platinum-cobalt Standard Method. The } \\
\text { DR/2000 instrument was used. Nkrumah (2011) }\end{array}$ \\
\hline 2. & Odour & Odour was determined through the sensation of smell. \\
\hline 3. & Temperature & This was measure using thermometer. \\
\hline 4. & $\mathrm{pH}$ & $\begin{array}{l}\text { Water } \mathrm{pH} \text { was determined using a Digital } \mathrm{pH} \text { meter. The meter was } \\
\text { calibrated using different buffer solutions of } \mathrm{pH} 12.1,10.1,7.0 \text { and } 4 \text {. }\end{array}$ \\
\hline 5. & $\begin{array}{l}\text { Electrical } \\
\text { Conductivity }\end{array}$ & $\begin{array}{l}\text { A Hatch conductivity meter } 4600 \text { was used to determine electrical } \\
\text { conductivity. An electrode connected to a meter was immersed into } \\
\text { the sample of water so that the water covered a sensitized electrode. } \\
\text { A stabilized value on the display was obtained and recorded. }\end{array}$ \\
\hline 6. & Turbidity & $\begin{array}{l}\text { This was determined using a potable turbidity meter. The meter was } \\
\text { calibrated by standardizing with distilled water and the sample } \\
\text { placed inside the cell holder. }\end{array}$ \\
\hline 7. & Total Hardness & $\begin{array}{c}\text { Total hardness was analyzed by titration of } 50 \mathrm{ml} \text { water sample with } \\
\text { standard EDTA }\end{array}$ \\
\hline 8. & $\begin{array}{l}\text { Dissolve } \\
\text { Oxygen }\end{array}$ & $\begin{array}{c}\text { Dissolved oxygen was measured titrimetrically using Winkler's } \\
\text { method after } 5 \text { days incubation at } 293 \mathrm{~K}\end{array}$ \\
\hline 9. & Nitrate & $\begin{array}{l}\text { This was measured by direct reading of spectrophotometer at } 425 \\
\text { nm radiation by making a colour complex with Nessler's reagent. }\end{array}$ \\
\hline
\end{tabular}




\begin{tabular}{|c|c|c|}
\hline $\mathbf{S} / \mathbf{N}$ & Test & Method and Procedures \\
\hline 10. & Chlorine & $\begin{array}{l}\text { It was measured by titrating a known volume of sample with } \\
\text { standardized silver nitrate solution using potassium chromate } \\
\text { solution in water or eosin/fluorescein solution in alcohol as } \\
\text { indicator. }\end{array}$ \\
\hline 11. & Iron & $\begin{array}{l}\text { It was measured through a colour changing test strip kit to detect the } \\
\text { level of iron in the water. }\end{array}$ \\
\hline 12. & $\begin{array}{l}\text { Total Bacteria } \\
\text { Count }\end{array}$ & $\begin{array}{l}\text { Total heterotrophic bacteria in the water samples were obtained } \\
\text { using the pour plate method. }\end{array}$ \\
\hline 13. & $\begin{array}{l}\text { Total Coliform } \\
\text { Count }\end{array}$ & $\begin{array}{l}\text { The enumeration and isolation of coliform bacteria was by the use of } \\
\text { the membrane filtration technique (Eckner, 1998; Jagalset al., 2000) } \\
\text { and growth on MacConkey agar. }\end{array}$ \\
\hline 14. & E-coli & $\begin{array}{l}\text { The presence of Escherichia coli in the water samples was assessed } \\
\text { by growth and colour reaction on Eosin }\end{array}$ \\
\hline
\end{tabular}

Table 1: Laboratory Analysis

Source: (APHA 1985, Degrandpre 1993, APHA 1998, Trivedy, andGoal 1986, Kodarkar1992, Wang 2002, ASTM 2003, Owolabi, Et Al, 2014, Akpen, Et Al., 2018)

\section{Results and Discussion}

\subsection{The Physiochemical and Biological Properties of Sachet Water in MubiMetropolis, Adamawa State}

During the first week of production as shown in table 2, all sachet water sampled was colorless and odorless. The temperatures of all the sachet water sampled were homogenous and ranges between $27.30^{\circ} \mathrm{C}$ and $27.60^{\circ} \mathrm{C}$, with an insignificant variation of $0.35 \%$. The $\mathrm{pH}$ ranges from 7.10 to 8.20 and have a mean of 7.59 , with Mugulbu Water having the highest $\mathrm{pH}$ value at 8.20 while AdsuMubi water and Whorawater have the lowest $\mathrm{pH}$ of 7.10. The pH of the sample sachet water exhibited an insignificant variability of 5.01\% (table 2).

\begin{tabular}{|c|c|c|c|c|c|c|c|c|c|c|c|c|c|}
\hline & $\sum_{\tilde{\omega}}^{\bar{n}}$ & 胥 & 占 & 趈 & 䣽 & 触 & 点 & 炭 & 葛 & 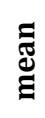 & 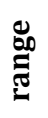 & की & $\begin{array}{l}d^{\circ} \\
d^{2}\end{array}$ \\
\hline & 禀 & 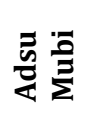 & $\sum_{\substack{\pi \\
\Sigma}}^{0}$ & 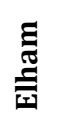 & $\frac{\pi}{3}$ & 莺 & z & & & & & & \\
\hline colour & $\begin{array}{l}\frac{\tilde{u}}{0} \\
\frac{0}{\tilde{0}} \\
0 \\
0\end{array}$ & $\begin{array}{l}\frac{\tilde{u}}{0} \\
\frac{0}{\tilde{0}} \\
0 \\
0\end{array}$ & $\begin{array}{l}\frac{u}{0} \\
\frac{0}{0} \\
\frac{0}{0} \\
0\end{array}$ & $\begin{array}{l}\frac{\tilde{u}}{0} \\
\frac{0}{\vdots} \\
\frac{0}{0}\end{array}$ & $\begin{array}{l}\frac{\tilde{u}}{0} \\
\frac{0}{0} \\
\frac{0}{0}\end{array}$ & $\begin{array}{l}\frac{u}{0} \\
\frac{d}{\square} \\
\frac{0}{0}\end{array}$ & $\frac{\mathscr{0}}{\frac{0}{0}}$ & 1 & 1 & 1 & 1 & I & \\
\hline Odour & 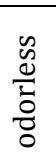 & 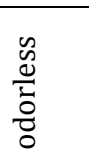 & $\begin{array}{l}\frac{w}{0} \\
\frac{0}{\tilde{0}} \\
\frac{0}{0}\end{array}$ & 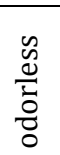 & $\begin{array}{l}\tilde{0} \\
\frac{\omega}{\tilde{0}} \\
\frac{0}{0}\end{array}$ & 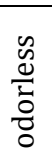 & $\begin{array}{l}\frac{w}{0} \\
\frac{0}{2} \\
\frac{\tilde{c}}{0}\end{array}$ & 1 & 1 & I & 1 & ' & \\
\hline $\begin{array}{c}\text { Temperature } \\
(0 \mathrm{c})\end{array}$ & $\stackrel{+}{\stackrel{N}{N}}$ & 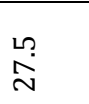 & $\stackrel{m}{\stackrel{n}{N}}$ & $\stackrel{+}{\stackrel{\sim}{N}}$ & 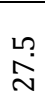 & $\stackrel{\stackrel{\sim}{\sim}}{\stackrel{N}{N}}$ & $\underset{\sim}{\sim}$ & $\begin{array}{l}\stackrel{8}{\grave{N}} \\
\stackrel{N}{N}\end{array}$ & 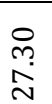 & 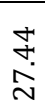 & $\begin{array}{l}m \\
0 \\
0\end{array}$ & $\begin{array}{l}0 \\
0 \\
0\end{array}$ & $\stackrel{n}{m}$ \\
\hline $\mathrm{pH}$ & $\begin{array}{c}\stackrel{N}{1} \\
\infty\end{array}$ & $\stackrel{\circ}{\stackrel{2}{n}}$ & $\stackrel{?}{\stackrel{2}{r}}$ & $\stackrel{\circ}{\stackrel{9}{r}}$ & $\stackrel{ }{\sim}$ & $\frac{\circ}{\circ}$ & $\stackrel{?}{\stackrel{2}{r}}$ & $\underset{\infty}{\stackrel{\infty}{\infty}}$ & $\stackrel{\circ}{\sim}$ & นิ & 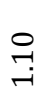 & $\stackrel{\infty}{\dddot{m}}$ & bे \\
\hline $\begin{array}{c}\text { Electrical } \\
\text { conductivity } \\
\text { (ms/cms) }\end{array}$ & $\begin{array}{l}\tilde{0} \\
\text { o. }\end{array}$ & $\stackrel{\mathscr{N}}{0}$ & $\stackrel{+}{:}$ & $\stackrel{n}{?}$ & $\stackrel{20}{\circ}$ & $\stackrel{\infty}{\circ}$ & సี & $\stackrel{\text { N̦}}{0}$ & $\stackrel{\sim}{0}$ & $\stackrel{m}{0}$ & సֶ. & $\stackrel{\circ}{\circ}$ & نें \\
\hline Turbidity & $\stackrel{\vec{N}}{0}$ & Ñ & $\stackrel{\widetilde{N}}{0}$ & $\stackrel{\infty}{\stackrel{\infty}{0}}$ & m. & นิ & m. & นิ & तี & m. & 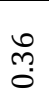 & సै & $\sqrt{-}$ \\
\hline $\begin{array}{c}\text { Total } \\
\text { Hardness } \\
\text { (mg/l }\end{array}$ & $\begin{array}{l}\text { ¿ } \\
\text { ț } \\
\text { İ }\end{array}$ & $\begin{array}{l}8 \\
\stackrel{+}{\infty}\end{array}$ & $\begin{array}{l}8 \\
\circ \\
0 \\
0\end{array}$ & $\begin{array}{l}8 \\
0 \\
\circ \\
\sigma\end{array}$ & $\begin{array}{l}\stackrel{8}{\circ} \\
\dot{\infty}\end{array}$ & $\begin{array}{l}\stackrel{0}{0} \\
\stackrel{i}{\sim} \\
\text {. }\end{array}$ & 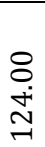 & 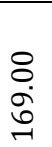 & $\begin{array}{l}\stackrel{8}{\circ} \\
\dot{\infty}\end{array}$ & 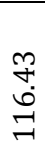 & 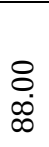 & $\begin{array}{l}2 \\
\infty \\
0 \\
\dot{m} \\
m\end{array}$ & เค \\
\hline
\end{tabular}




\begin{tabular}{|c|c|c|c|c|c|c|c|c|c|c|c|c|c|}
\hline & 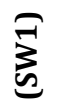 & 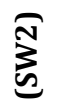 & 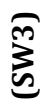 & $\underbrace{\frac{F}{5}}$ & $\underbrace{\frac{10}{5}}$ & $\underbrace{0}_{0}$ & 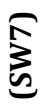 & $\stackrel{x}{\overparen{\Xi}}$ & $\Xi$ & ङ્ઁ & 范 & ติ & $e^{0}$ \\
\hline & 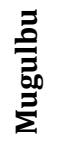 & 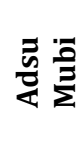 & 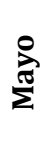 & 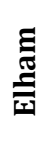 & $\overbrace{3}^{00}$ & 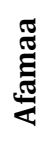 & 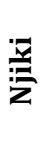 & & & & & & \\
\hline $\begin{array}{c}\text { Total } \\
\text { Dissolved } \\
\text { Solid (mg/l) }\end{array}$ & $\begin{array}{l}\stackrel{8}{0} \\
\stackrel{0}{0} \\
\stackrel{1}{*}\end{array}$ & $\begin{array}{l}8 \\
\stackrel{8}{+} \\
\end{array}$ & 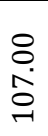 & $\begin{array}{l}\stackrel{8}{\circ} \\
\stackrel{-}{\rightrightarrows}\end{array}$ & $\begin{array}{l}\stackrel{8}{\circ} \\
\stackrel{+}{-} \\
\stackrel{-}{1}\end{array}$ & $\begin{array}{l}\stackrel{8}{ } \\
\text { ì }\end{array}$ & $\begin{array}{l}\text { ○ } \\
\text { ๙̇ }\end{array}$ & 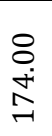 & $\begin{array}{l}\stackrel{8}{0} \\
\stackrel{0}{0} \\
\text { v }\end{array}$ & $\begin{array}{l}\circ \\
\infty \\
\circ\end{array}$ & \begin{tabular}{l}
8 \\
$\infty$ \\
$\infty$ \\
\multirow{+}{+}{}
\end{tabular} & $\stackrel{0}{\stackrel{0}{y}}$ & $\begin{array}{l}\stackrel{*}{n} \\
\stackrel{\leftrightarrow}{0} \\
\ddot{+}\end{array}$ \\
\hline $\begin{array}{l}\text { Dissolved } \\
\text { Oxygen } \\
\text { (mg/l) }\end{array}$ & $\begin{array}{l}8 \\
8 \\
0\end{array}$ & $\begin{array}{l}\stackrel{8}{8} \\
\stackrel{N}{N}\end{array}$ & $\begin{array}{l}8 \\
\infty \\
\infty \\
0\end{array}$ & $\begin{array}{l}8 \\
\stackrel{0}{0}\end{array}$ & $\begin{array}{l}\circ \\
\text { o̊ }\end{array}$ & $\begin{array}{l}8 \\
\infty \\
\infty \\
\infty\end{array}$ & $\begin{array}{l}\stackrel{\bigcirc}{0} \\
\end{array}$ & 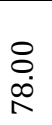 & $\begin{array}{l}8 \\
\infty \\
\infty \\
0\end{array}$ & ָָ & $\begin{array}{l}8 \\
\stackrel{0}{0} \\
\stackrel{-}{-1}\end{array}$ & $\stackrel{\sigma}{\vec{m}}$ & $\begin{array}{l}\infty \\
\stackrel{+}{+}\end{array}$ \\
\hline $\begin{array}{l}\text { Nitrate } \\
(\mathrm{mg} / \mathrm{l})\end{array}$ & $\begin{array}{l}\stackrel{\circ}{\circ} \\
\stackrel{0}{\infty} \\
\stackrel{N}{ }\end{array}$ & $\begin{array}{l}\circ \\
\stackrel{0}{0} \\
0\end{array}$ & $\begin{array}{l}\text { 웅 } \\
\text { ㄱ. }\end{array}$ & 용 & $\begin{array}{l}\circ \\
\stackrel{0}{0} \\
0\end{array}$ & $\begin{array}{l}\stackrel{8}{0} \\
\stackrel{1}{\rightarrow}\end{array}$ & 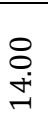 & 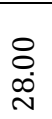 & $\begin{array}{l}\circ \\
\stackrel{0}{0} \\
0 \\
\stackrel{-}{1}\end{array}$ & $\begin{array}{l}\text { ำ } \\
\text { + }\end{array}$ & $\begin{array}{l}8 \\
\infty \\
\infty \\
\infty\end{array}$ & $\begin{array}{l}\text { ผ } \\
\text { ผ่ }\end{array}$ & $\begin{array}{l}{ }_{+}^{*} \\
\infty \\
\dot{+}\end{array}$ \\
\hline $\begin{array}{c}\text { Chlorine } \\
(\mathrm{mg} / \mathrm{l})\end{array}$ & 울 & $\begin{array}{l}\stackrel{8}{0} \\
\stackrel{0}{0}\end{array}$ & ○े & $\begin{array}{l}\stackrel{8}{\circ} \\
\stackrel{-}{-1}\end{array}$ & $\begin{array}{l}\stackrel{8}{\circ} \\
\stackrel{+}{N}\end{array}$ & $\begin{array}{l}\stackrel{8}{ } \\
\dot{m}\end{array}$ & $\begin{array}{l}\stackrel{\circ}{\circ} \\
\circ \\
\stackrel{-}{-}\end{array}$ & $\begin{array}{l}\stackrel{8}{0} \\
\stackrel{\leftrightarrow}{\sim}\end{array}$ & $\begin{array}{l}8 \\
\text { เi }\end{array}$ & 壱 & $\begin{array}{l}\stackrel{\circ}{\circ} \\
\stackrel{i}{N}\end{array}$ & ํㅜㄴ & $\begin{array}{l}* \\
\infty \\
\infty \\
\infty \\
\infty \\
+\end{array}$ \\
\hline Iron (mg/) & $\stackrel{m}{m}$ & $\stackrel{m}{0}$ & $\vec{~}$ & $\stackrel{\circ}{\stackrel{2}{0}}$ & $\stackrel{+}{0}$ & $\begin{array}{l}\text { Lo } \\
0 \\
0\end{array}$ & $\begin{array}{l}0 \\
0 \\
0\end{array}$ & $\stackrel{ }{\stackrel{-}{0}}$ & mo & $\begin{array}{l}0 \\
0 \\
0\end{array}$ & $\begin{array}{l}\infty \\
0 \\
0\end{array}$ & $\stackrel{m}{0}$ & $\begin{array}{l}\text { * } \\
\text { Oे } \\
0 \\
\text { ○े }\end{array}$ \\
\hline $\begin{array}{c}\text { Total } \\
\text { Bacterial } \\
(\mathrm{Cfu} / \mathrm{mol})\end{array}$ & $\begin{array}{c}\stackrel{0}{7} \\
\stackrel{*}{+} \\
\stackrel{i}{N}\end{array}$ & $\begin{array}{l}0 \\
\stackrel{*}{*} \\
\infty \\
\rightarrow \\
\rightarrow\end{array}$ & $\begin{array}{l}\stackrel{0}{\overrightarrow{1}} \\
\stackrel{*}{\wedge} \\
\stackrel{\sim}{v}\end{array}$ & $\begin{array}{l}\stackrel{0}{\vec{H}} \\
\stackrel{*}{\mathrm{~L}} \\
\mathrm{~N}\end{array}$ & $\begin{array}{l}\stackrel{0}{\vec{*}} \\
\stackrel{*}{\vec{n}} \\
\dot{m}\end{array}$ & $\begin{array}{l}\stackrel{0}{-1} \\
\stackrel{*}{\sigma} \\
\stackrel{j}{j}\end{array}$ & $\begin{array}{l}\stackrel{\circ}{\vec{H}} \\
\stackrel{*}{m} \\
\stackrel{N}{N}\end{array}$ & $\begin{array}{l}\stackrel{0}{\overrightarrow{1}} \\
\stackrel{*}{\stackrel{-}{n}} \\
\dot{m}\end{array}$ & $\begin{array}{l}0 \\
\stackrel{7}{*} \\
\infty \\
\infty \\
\rightarrow\end{array}$ & $\begin{array}{l}\stackrel{0}{7} \\
\stackrel{*}{*} \\
\text { in }\end{array}$ & $\begin{array}{l}\stackrel{0}{7} \\
\stackrel{*}{m} \\
\rightarrow\end{array}$ & $\begin{array}{l}0 \\
0 \\
0\end{array}$ & 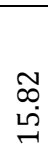 \\
\hline $\begin{array}{c}\text { Total } \\
\text { Coliform } \\
\text { Count } \\
(\mathrm{Cfu} / \mathrm{mol})\end{array}$ & $\begin{array}{c}\stackrel{0}{7} \\
\stackrel{*}{m} \\
\stackrel{i}{N}\end{array}$ & $\begin{array}{l}\stackrel{0}{\overrightarrow{1}} \\
\stackrel{*}{6} \\
\stackrel{i}{N}\end{array}$ & $\begin{array}{l}\stackrel{0}{7} \\
\stackrel{*}{6} \\
\stackrel{-}{-1}\end{array}$ & $\begin{array}{l}\stackrel{0}{*} \\
\stackrel{*}{+} \\
\dot{m}\end{array}$ & 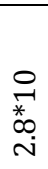 & $\begin{array}{l}\stackrel{0}{1} \\
\text { * } \\
\text { Ln } \\
\text { N }\end{array}$ & \begin{tabular}{l}
$\stackrel{0}{7}$ \\
\multirow{*}{*}{} \\
$\stackrel{0}{0}$ \\
Ni
\end{tabular} & $\begin{array}{l}\stackrel{0}{\overrightarrow{1}} \\
\stackrel{*}{+} \\
\dot{m}\end{array}$ & $\begin{array}{l}\stackrel{0}{7} \\
\underset{*}{6} \\
\stackrel{-}{-1}\end{array}$ & $\begin{array}{l}\stackrel{0}{7} \\
\underset{*}{0} \\
\stackrel{i}{i}\end{array}$ & $\begin{array}{l}0 \\
\underset{*}{*} \\
\infty \\
\end{array}$ & 오 & $\begin{array}{l}m \\
m \\
\sim\end{array}$ \\
\hline $\begin{array}{c}\text { E-Coli } \\
(\mathrm{Cfu} / \mathrm{mol})\end{array}$ & $\begin{array}{l}8 \\
\circ \\
\circ\end{array}$ & $\begin{array}{l}\stackrel{0}{0} \\
\dot{0}\end{array}$ & : & : & : & $\begin{array}{l}8 \\
0 \\
0\end{array}$ & $\begin{array}{l}\circ \\
\circ \\
\circ\end{array}$ & $\begin{array}{l}8 \\
\circ \\
\circ\end{array}$ & $\begin{array}{l}\circ \\
\stackrel{0}{0}\end{array}$ & $\begin{array}{l}8 \\
\circ\end{array}$ & $\begin{array}{l}8 \\
\circ\end{array}$ & 1 & 1 \\
\hline
\end{tabular}

Table 2: Physiochemical and Biological Properties of Sachet Water in MubiMetropolis, Adamawa State (Week 1) $S D=$ Standard Deviation, $C V=$ Coefficient of Variation, Significant C.V $\geq 33 \%$

Source: Author's Compilation (2021)

The electrical conductivity of the sachet water ranges from $0.02 \mathrm{~ms} / \mathrm{cm}^{3}$ to $0.23 \mathrm{~ms} / \mathrm{cm}^{3}$ and exhibited a significant variation of $46.15 \%$ (see table 2). AdsuMubi exhibited the highest EC value at 0.23 while MugulbuWater exhibited the lowest at $0.02 \mathrm{~ms} / \mathrm{cm}^{3}$. The EC value of AdsuMubi and Mugulbu Water may be attributed directly to their level of total dissolved solid (Isikwu and Chikezie, 2014). The turbidity of the sampled sachet water ranges from 0.21NTU to 0.57NTU, with a mean value of $0.31 \mathrm{NTU}$ and significant variation at $38.71 \%$ (see table 2 ). Mayo Water exhibited the highest turbidity level of $0.57 \mathrm{NTU}$ while Mugulbu Water exhibited the lowest turbidity level of $0.21 \mathrm{NTU}$. The generally low turbidity values observed in the samples are in line with the physical observation of the samples, which revealed odourless, clear and colourless samples.The total hardness of the samples ranges from $81 \mathrm{mg} / \mathrm{l}$ to $169 \mathrm{mg} / \mathrm{l}$, with a mean of $116.43 \mathrm{mg} / \mathrm{l}$ and an insignificant variation of $26.50 \%$ (see table 2). Elhamwas observed to have the highest total hardness level at $169 \mathrm{~g} / \mathrm{l}$ while Whora have the lowest at $81 \mathrm{mg} / \mathrm{l}$. The TDS content of the sampled sachet water ranges between $26.00 \mathrm{mg} / \mathrm{l}$ and $174 \mathrm{mg} / \mathrm{l}$.

With a mean, range and significant CV value of $98.00 \mathrm{mg} / \mathrm{l}, 148.00 \mathrm{mg} / \mathrm{l}$ and $43.63 \%$ respectively (see table 2 ). AdsuMubi exhibited the highest TDS content of $174 \mathrm{mg} / \mathrm{l}$ while Mugulbu Water indicated the lowest at 26.00mg/l. Consequently, AdsuMubi exhibited the highest EC level while Mugulbu water exhibited the lowest EC level on the other hand. According to Isikwue and Chikezie (2014), the TDS level in all of the sachet water may be attributable to the salt concentration from the basement formation from where the water packaged comes from.Dissolved oxygen content of the sampled sachet water is relatively homogenous with a CV value of just $4.48 \%$ and ranges from $68.00 \mathrm{mg} / \mathrm{l}$ to $78 \mathrm{mg} / \mathrm{l}$ (see table 4.3.1). Elhamindicated the lowest content at $68.00 \mathrm{mg} / \mathrm{l}$ while Mayo Water indicated the highest value at $78.00 \mathrm{mg} / \mathrm{l}$.The nitrate content of the sampled sachet water ranges from $10.00 \mathrm{mg} / \mathrm{l}$ to $28.00 \mathrm{mg} / \mathrm{l}$, with a mean value of $14.57 \mathrm{mg} / \mathrm{l}$ and a significant variation at $40.84 \%$ (see table 2). Mugulbu Water exhibited the highest nitrate content of $28.00 \mathrm{mg} / \mathrm{l}$ while AdsuMubi and Whoraexhibited the lowest nitrate content of $10.00 \mathrm{mg} / \mathrm{l}$. 
The chlorine content of the sampled sachet water ranges between $5.00 \mathrm{mg} / \mathrm{l}$ and $26.00 \mathrm{mg} / \mathrm{l}$, with a mean value of $15.14 \mathrm{mg} / \mathrm{l}$, range of $21.00 \mathrm{mg} / \mathrm{l}$ and a significant variation at $48.88 \%$ (see table 2). The high range and CV value indicate the high heterogeneity of chlorine content in the sampled sachet water, which may be attributed to the sources of water, treatment methods, packaging deficiencies (Isikwue, and Chikezie, 2014; WHO, 2014; Akpan,et al,2018). The iron content of water is of considerable concern because small amounts can seriously affect its suitability for drinking and colour. The iron content of the sampled sachet water ranges from $0.03 \mathrm{mg} / \mathrm{l}$ to $0.11 \mathrm{mg} / \mathrm{l}$ and exhibited a significant variation of $50.00 \%$ (see table 2). The highest iron content was observed in Elhamwhile Mugulbu Water and AdsuMubi have the lowest iron content. The total Bacteria Count (TBC) of the sampled sachet water ranges between $1.8^{*} 10^{1}$ and $3.1^{*} 10^{1}$, with a mean and $\mathrm{CV}$ of $2.5^{*} 10^{1}$ and $15.82 \%$ respectively. Whora has the highest TBC content of $3.1^{*} 10^{1}$ while AdsuMubi has the lowest at $1.8^{*} 10^{1}$. Similarly, Total Coliform Count (TCC) ranges between $1.6^{*} 10^{1}$ and $3.4^{*} 10^{1}$, with a mean and CV of $2.6^{*} 10^{1}$ and $19.33 \%$ respectively. On the other hand, E.coli is absent in all of the sampled sachet water (table 2).

\begin{tabular}{|c|c|c|c|c|c|c|c|c|c|c|c|c|c|}
\hline & $\sum^{3}$ & 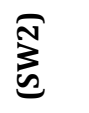 & 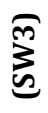 & 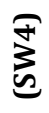 & 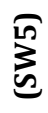 & $\sum^{0}$ & 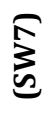 & 竎 & $\Xi$ & $\stackrel{\Xi}{\Xi}$ & 兽 & के & $\frac{a}{d}$ \\
\hline$\frac{\vdots}{0}$ & ڤ્ટ & 预 & 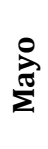 & Е & $\frac{\pi}{3}$ & $\underset{\mathbb{Z}}{\mathbb{Z}}$ & 竞 & & & & & & \\
\hline Odour & $\frac{\mathscr{d}}{\stackrel{0}{\pi}}$ & $\frac{\mathscr{n}}{\stackrel{0}{\check{I}}}$ & $\frac{\mathscr{d}}{\stackrel{\mathscr{d}}{\pi}}$ & $\frac{\mathscr{y}}{\frac{\tilde{d}}{\tilde{D}}}$ & $\frac{\tilde{d}}{\frac{\tilde{d}}{\tilde{0}}}$ & $\frac{\tilde{d}}{\stackrel{0}{\tilde{0}}}$ & $\frac{\mathscr{d}}{\frac{\tilde{d}}{\tilde{D}}}$ & I & 1 & 1 & 1 & 1 & I \\
\hline $\begin{array}{c}\text { Temperature } \\
\text { (0c) }\end{array}$ & $\begin{array}{l}\frac{w}{0} \\
\frac{0}{\pi} \\
\frac{0}{0}\end{array}$ & $\frac{\mathscr{n}}{\stackrel{d}{\pi}}$ & $\frac{\mathscr{y}}{0}$ & $\begin{array}{l}\mathscr{y} \\
\frac{0}{1} \\
\frac{0}{0} \\
0\end{array}$ & $\frac{\mathscr{d}}{\frac{0}{2}}$ & 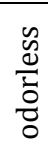 & $\frac{\mathscr{w}}{\frac{0}{\pi}}$ & 1 & 1 & 1 & 1 & 1 & 1 \\
\hline $\mathrm{pH}$ & $\stackrel{⿱ 亠 凶}{N}$ & $\stackrel{\text { ำ }}{\stackrel{N}{N}}$ & $\stackrel{m}{N}$ & $\stackrel{+}{\stackrel{\sim}{N}}$ & $\stackrel{\text { ᄂ? }}{\stackrel{N}{N}}$ & $\stackrel{t}{\stackrel{\sim}{N}}$ & $\stackrel{0}{N}$ & $\begin{array}{l}\text { ○ } \\
\text { ஸे }\end{array}$ & $\stackrel{\circ}{\text { ñ }}$ & 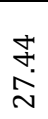 & $\stackrel{m}{0}$ & o̊ & 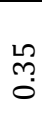 \\
\hline $\begin{array}{c}\text { Electrical } \\
\text { conductivity }\end{array}$ & $\stackrel{\substack{n \\
\infty}}{\infty}$ & 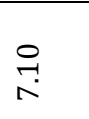 & $\frac{9}{1}$ & ํㅜㅁ & $\stackrel{゚}{N}$ & 赵 & $\stackrel{0}{i}$ & 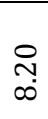 & $\stackrel{ }{\stackrel{ }{r}}$ & ำ & 욱. & $\stackrel{\infty}{m}$ & نे \\
\hline Turbidity & $\begin{array}{l}\text { Oे } \\
0\end{array}$ & $\stackrel{n}{i}$ & $\stackrel{+}{\breve{a}}$ & $\stackrel{\text { Ln }}{\stackrel{0}{0}}$ & $\stackrel{\text { Ln }}{\stackrel{2}{0}}$ & $\begin{array}{l}\infty \\
0 \\
0\end{array}$ & $\stackrel{?}{0}$ & 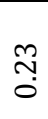 & ֻै & $\stackrel{m}{\stackrel{0}{0}}$ & $\stackrel{\check{N}}{\sigma}$ & $\begin{array}{l}0 \\
0 \\
0\end{array}$ & 苗 \\
\hline $\begin{array}{c}\text { Total } \\
\text { Hardness } \\
\text { (mg/l }\end{array}$ & $\stackrel{\check{N}}{0}$ & $\stackrel{N}{0}$ & $\underset{N}{\tilde{\sigma}}$ & $\stackrel{\substack{N \\
0}}{0}$ & m़े & ํํํ & $\ddot{n}$ & ำ & $\stackrel{\check{N}}{\sigma}$ & $\ddot{m}$ & $\stackrel{0}{m}$ & $\stackrel{N}{3}$ & 空 \\
\hline $\begin{array}{c}\text { Total } \\
\text { Dissolved } \\
\text { Solid (mg/l) }\end{array}$ & 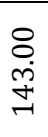 & $\begin{array}{l}\text { \& } \\
\text { L் } \\
\infty\end{array}$ & $\begin{array}{l}\text { \& } \\
\text { îj } \\
\text {-1 }\end{array}$ & $\begin{array}{l}8 \\
\text { व் }\end{array}$ & 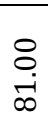 & 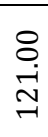 & 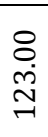 & $\begin{array}{l}8 \\
0 \\
0 \\
0 \\
-1\end{array}$ & @ & $\begin{array}{l}\stackrel{m}{+} \\
\stackrel{0}{-}\end{array}$ & $\begin{array}{l}8 \\
\infty \\
\infty\end{array}$ & $\begin{array}{l}\text { Ln } \\
\infty \\
\stackrel{\infty}{\infty}\end{array}$ & $\begin{array}{l}\text { 울 } \\
\text { ஸุ } \\
\text { N }\end{array}$ \\
\hline $\begin{array}{l}\text { Dissolved } \\
\text { Oxygen } \\
\text { (mg/l) }\end{array}$ & $\begin{array}{l}8 \\
\text { Oे } \\
\stackrel{1}{N}\end{array}$ & 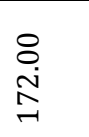 & $\begin{array}{l}8 \\
0 \\
0 \\
0\end{array}$ & $\begin{array}{l}8 \\
\stackrel{0}{0} \\
\stackrel{-}{1}\end{array}$ & $\begin{array}{l}\stackrel{8}{0} \\
\stackrel{m}{=}\end{array}$ & $\begin{array}{l}8 \\
\text { iु }\end{array}$ & ஃ̊ & 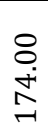 & $\begin{array}{l}8 \\
\stackrel{0}{0} \\
\stackrel{0}{N}\end{array}$ & $\begin{array}{l}\text { ○ } \\
\infty \\
\curvearrowleft\end{array}$ & $\begin{array}{l}8 \\
\stackrel{0}{\infty} \\
\stackrel{\infty}{+} \\
+\end{array}$ & 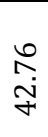 & $\begin{array}{l}\stackrel{*}{n} \\
\stackrel{\leftrightarrow}{+}\end{array}$ \\
\hline $\begin{array}{l}\text { Nitrate } \\
(\mathrm{mg} / \mathrm{l})\end{array}$ & $\begin{array}{l}8 \\
\infty \\
\infty \\
0\end{array}$ & 욤 & @ & 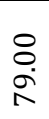 & $\begin{array}{l}\circ \\
\text { ஸ̂ }\end{array}$ & $\begin{array}{l}8 \\
\text { م } \\
\end{array}$ & $\begin{array}{l}\text { ○ } \\
\text { ホ̦ }\end{array}$ & $\begin{array}{l}\bigcirc \\
0 \\
\infty \\
\end{array}$ & $\begin{array}{l}\circ \\
0 \\
\infty \\
0\end{array}$ & ָָ & $\begin{array}{l}\circ \\
\stackrel{0}{0} \\
\stackrel{1}{-1}\end{array}$ & $\underset{\sim}{\stackrel{\sigma}{n}}$ & $\stackrel{\infty}{+}$ \\
\hline $\begin{array}{c}\text { Chlorine } \\
\text { (mg/l) }\end{array}$ & $\begin{array}{l}8 \\
\text { ํ. } \\
\text { ה }\end{array}$ & $\begin{array}{l}\stackrel{8}{0} \\
\stackrel{\text { I }}{-}\end{array}$ & $\begin{array}{l}8 \\
0 \\
0 \\
-1\end{array}$ & $\begin{array}{l}8 \\
\stackrel{-}{\circ} \\
\stackrel{-}{1}\end{array}$ & $\begin{array}{l}\stackrel{8}{0} \\
\stackrel{0}{0} \\
\stackrel{-1}{0}\end{array}$ & $\begin{array}{l}\stackrel{8}{0} \\
\text { ㄱ } \\
\text { I }\end{array}$ & $\begin{array}{l}\stackrel{8}{0} \\
\dot{m} \\
\rightarrow\end{array}$ & $\begin{array}{l}\text { ○ } \\
\infty \\
\stackrel{0}{N}\end{array}$ & $\begin{array}{l}0 \\
\stackrel{0}{0} \\
\stackrel{0}{-}\end{array}$ & \begin{tabular}{l} 
in \\
\multirow{+}{+}{}
\end{tabular} & $\begin{array}{l}8 \\
\varnothing \\
\infty \\
\rightarrow\end{array}$ & $\begin{array}{l}\text { 오 } \\
\text { ผ่ }\end{array}$ & 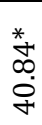 \\
\hline Iron $(\mathrm{mg} /$ ) & $\begin{array}{l}8 \\
\stackrel{\leftrightarrow}{\sim} \\
\stackrel{+}{1}\end{array}$ & $\begin{array}{l}\stackrel{8}{ } \\
\stackrel{+}{N}\end{array}$ & $\begin{array}{l}8 \\
\text { ă }\end{array}$ & $\begin{array}{l}\stackrel{8}{ } \\
\stackrel{\text { J }}{\text { D }}\end{array}$ & $\begin{array}{l}\text { ○ } \\
\infty \\
\stackrel{0}{-1}\end{array}$ & ○. & $\begin{array}{l}\stackrel{8}{0} \\
\stackrel{+}{\circ}\end{array}$ & $\begin{array}{l}8 \\
0 \\
\stackrel{0}{N}\end{array}$ & $\begin{array}{l}8 \\
\text { ¿ }\end{array}$ & $\begin{array}{l}\overrightarrow{+} \\
\text { เ̇ }\end{array}$ & 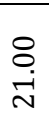 & 운 & $\begin{array}{l}* \\
\infty \\
\infty \\
\infty \\
\infty \\
+\end{array}$ \\
\hline $\begin{array}{c}\text { Total } \\
\text { Bacterial } \\
\text { count } \\
(\mathrm{Cfu} / \mathrm{mol})\end{array}$ & $\stackrel{1}{\mathfrak{a}}$ & $\stackrel{m}{0}$ & $\begin{array}{l}\text { Oे } \\
0\end{array}$ & $\stackrel{0}{\stackrel{-}{0}}$ & 농 & $\begin{array}{l}0 \\
0 \\
0\end{array}$ & $\begin{array}{l}0 \\
\stackrel{0}{0}\end{array}$ & $\stackrel{-1}{\stackrel{-}{0}}$ & $\stackrel{m}{0}$ & $\begin{array}{l}0 \\
0 \\
0\end{array}$ & $\begin{array}{l}\infty \\
0 \\
0\end{array}$ & $\begin{array}{l}m \\
0 \\
0\end{array}$ & 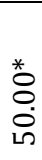 \\
\hline
\end{tabular}




\begin{tabular}{|c|c|c|c|c|c|c|c|c|c|c|c|c|c|}
\hline & 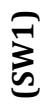 & 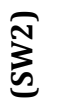 & 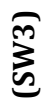 & $\underbrace{\frac{F}{5}}$ & $\underbrace{\frac{10}{5}}$ & $\underbrace{0}$ & 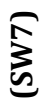 & 苂 & $\Xi$ & $\stackrel{\Xi}{\Xi}$ & 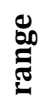 & ) & $\underbrace{a}_{3}$ \\
\hline$\frac{3}{8}$ & 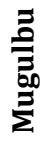 & 突完 & 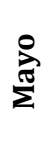 & 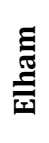 & $\frac{\pi}{3}$ & 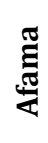 & 䆓 & & & & & & \\
\hline $\begin{array}{c}\text { Total } \\
\text { Coliform } \\
\text { Count } \\
(\mathrm{Cfu} / \mathrm{mol})\end{array}$ & $\begin{array}{l}\stackrel{0}{\rightarrow} \\
\stackrel{*}{\oplus} \\
\text { N }\end{array}$ & 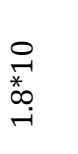 & $\begin{array}{l}\stackrel{0}{\overrightarrow{1}} \\
\stackrel{*}{\mathrm{~L}} \\
\text { N }\end{array}$ & $\begin{array}{l}\stackrel{0}{-1} \\
\stackrel{*}{n} \\
\stackrel{n}{v}\end{array}$ & $\begin{array}{l}0 \\
\stackrel{+}{*} \\
\stackrel{0}{0} \\
\dot{m}\end{array}$ & $\begin{array}{l}0 \\
\stackrel{+}{*} \\
\infty \\
\stackrel{\infty}{N}\end{array}$ & $\begin{array}{l}\stackrel{ }{\overrightarrow{1}} \\
\stackrel{*}{\sim} \\
\underset{N}{n}\end{array}$ & $\begin{array}{l}\stackrel{0}{7} \\
\text { * } \\
\stackrel{0}{1} \\
\dot{m}\end{array}$ & 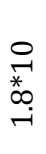 & $\begin{array}{l}\stackrel{0}{\vec{H}} \\
\stackrel{*}{+} \\
\stackrel{\sim}{\sim}\end{array}$ & 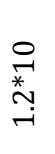 & ñ. & $\begin{array}{l}\text { mे } \\
\text { மn }\end{array}$ \\
\hline $\begin{array}{c}\text { E-Coli } \\
(\mathrm{Cfu} / \mathrm{mol})\end{array}$ & $\begin{array}{l}\stackrel{0}{\overrightarrow{1}} \\
\stackrel{*}{n} \\
\text { Niv }\end{array}$ & $\begin{array}{l}\stackrel{0}{\overrightarrow{1}} \\
\stackrel{*}{\stackrel{\text { v }}{*}}\end{array}$ & 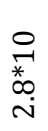 & $\begin{array}{l}\stackrel{0}{\overrightarrow{1}} \\
\stackrel{*}{L_{0}} \\
\text { m. }\end{array}$ & 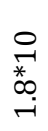 & $\begin{array}{l}\stackrel{0}{-1} \\
\stackrel{*}{*} \\
\text { Na }\end{array}$ & $\begin{array}{l}\stackrel{0}{7} \\
\text { 。. } \\
\text { iv }\end{array}$ & $\begin{array}{l}\stackrel{0}{\overrightarrow{1}} \\
\stackrel{*}{4} \\
\dot{m}\end{array}$ & $\begin{array}{l}\stackrel{0}{-1} \\
\stackrel{*}{6} \\
\stackrel{-1}{-1}\end{array}$ & $\begin{array}{l}\stackrel{0}{\overrightarrow{1}} \\
\underset{*}{6} \\
\stackrel{v}{v}\end{array}$ & $\begin{array}{l}\stackrel{0}{\overrightarrow{1}} \\
\underset{*}{\infty} \\
\stackrel{+}{\sim}\end{array}$ & 노 & $\begin{array}{l}m \\
m \\
\stackrel{\sim}{\sim}\end{array}$ \\
\hline & & $\stackrel{\circ}{\circ}$ & @ & @ & @ & ○. & $\stackrel{8}{\circ}$ & ○ & ○ & @ & : & 1 & I \\
\hline
\end{tabular}

Table 3 Physiochemical and Biological Properties of Sachet Water in MubiMetropolis, Adamawa State (Week 3) $S D=$ Standard Deviation, $C V=$ Coefficient of Variation, Significant C.V $\geq 33 \%$

Source: Author's Compilation (2021)

During the third week of production as shown in table 3, most of the physical properties of sampled sachet water remain fairly the same as the first week. The colour, odour, temperature, $\mathrm{pH}$, electrical conductivity and turbidity of all sampled sachet water remain fairly the same. The sampled sachet water remained colourless and odourless. The temperature of all the sachet water sampled remains fairly the same and homogenous, ranging from $27.30^{\circ} \mathrm{C}$ to $27.60^{\circ} \mathrm{C}$. The $\mathrm{pH}$ remains homogenous among all sample and ranges between 7.10 and 8.20, with a mean of 7.59, with Mugulbu Water having the highest $\mathrm{pH}$ value at 8.20 while AsduMubi and Whorahave the lowest pH of 7.10. Also, the electrical conductivity of the sachet water ranges from $0.02 \mathrm{~ms} / \mathrm{cm}^{3}$ to $0.23 \mathrm{~ms} / \mathrm{cm}^{3}$ and exhibited a significant variation of $46.15 \%$ just like the first week (see table 3). Similarly, the turbidity of the sampled sachet water ranges from $0.22 \mathrm{NTU}$ to $0.57 \mathrm{NTU}$, with a mean value of 0.31 NTU and significant variation at $38.71 \%$ (see table 3 ). However, while Mayo Water exhibited the highest turbidity level of 0.57NTU, Mugulbu Water and Elhamexhibited the lowest turbidity level of $0.22 \mathrm{NTU}$. The total hardness of the samples ranges from $81 \mathrm{mg} / \mathrm{l}$ to $162 \mathrm{mg} / \mathrm{l}$, with a mean of $115.00 \mathrm{mg} / \mathrm{l}$ and an insignificant variation of $24.97 \%$ (see table 3). Similar to the first week, Elhamwas observed to have the highest total hardness level at $162 \mathrm{mg} / \mathrm{l}$ while Whora have the lowest at $81 \mathrm{mg} / \mathrm{l}$. The Total Dissolved Solid (TDS) content ranges between $26.00 \mathrm{mg} / \mathrm{l}$ and $172 \mathrm{mg} / \mathrm{l}$. With a mean, range and significant CV value of $97.29 \mathrm{mg} / \mathrm{l}, 146.00 \mathrm{mg} / \mathrm{l}$ and $43.20 \%$ respectively (see table 3 ). AdsuMubi and Mugulbu Water still exhibited the highest and lowest TDS content respectively. On the other hand, the dissolved oxygen content of the sampled sachet water remained relatively homogenous but reduced slightly when compared to the first week and ranges from $68.00 \mathrm{mg} / \mathrm{l}$ to $77 \mathrm{mg} / \mathrm{l}$ (see table 3). Unlike the first week, Mugulbu Water and Elham indicated the lowest content at $68.00 \mathrm{mg} / \mathrm{l}$ while Mayo Water still indicated the highest value at $77.00 \mathrm{mg} / \mathrm{l}$.

The nitrate content of the sampled sachet water ranges from $10.00 \mathrm{mg} / \mathrm{l}$ to $27.00 \mathrm{mg} / \mathrm{l}$, with a mean value of $14.43 \mathrm{mg} / \mathrm{l}$ and a significant variation at 39.71\% (see table 3). Mugulbu Water exhibited the highest nitrate content of $27.00 \mathrm{mg} / \mathrm{l}$ while AdsuMubi and Whoraexhibited the lowest nitrate content of $10.00 \mathrm{mg} / \mathrm{l}$. The chlorine content of the sampled sachet water reduced slightly when compared to the first week. The observed chlorine content ranges between $5.00 \mathrm{mg} / \mathrm{l}$ and $24.00 \mathrm{mg} / \mathrm{l}$, with a reduced mean value of $14.43 \mathrm{mg} / \mathrm{l}$, range of $19.00 \mathrm{mg} / \mathrm{l}$ and a significant variation at $45.53 \%$ indicating heterogenic chlorine content within the sachet water (see table 3). Similarly, the iron content of the sampled sachet water ranges from $0.02 \mathrm{mg} / \mathrm{l}$ to $0.11 \mathrm{mg} / \mathrm{l}$ and exhibited a significant variation of $50.00 \%$ (see table 3 ). The sachet water with the highest iron content remains ElhamwhileMugulbu Water still exhibited the lowest iron content. The Total Coliform Count (TBC) of the sampled sachet water reduced slightly when compared to the first week of production and ranges between $1.8^{*} 10^{1}$ and $3.0^{*} 10^{1}$, with a mean and CV of $2.4^{*} 10^{1}$ and $15.34 \%$ respectively. In harmony with the result of the first week analysis, Whorahas the highest TBC content of $3.0^{*} 10^{1}$ while AsduMubi has the lowest of $1.8^{*} 10^{1}$. The Total Coliform Count (TCC) generally increased slightly when compared to the first week. The TCC ranges between $1.8^{*} 10^{1}$ and $3.5^{*} 10^{1}$, with a mean and CV of $2.6^{*} 10^{1}$ and $19.49 \%$ respectively. On the other hand, E.coli remained absent in all of the sampled sachet water (see table 3 ).

During the fifth week of production as shown in table 4, the physical properties of sampled sachet water remain fairly the same as the first and third week. The colour, odour, temperature, $\mathrm{pH}$, electrical conductivity and turbidity of all sampled sachet water remain fairly the same. This is similar to the findings of Martins and Ada (2014) that assess the quality of various sachet water brands marketed in Bauchi Metropolis of Nigeria. The sampled sachet water remained colourless and odourless. The temperature of all the sachet water sampled remains fairly the same and homogenous, ranging from $25.30^{\circ} \mathrm{C}$ to $25.60^{\circ} \mathrm{C}$. The $\mathrm{pH}$ remains homogenous among all sample and ranges between 7.10 and 8.20 , with a mean of 7.60. This implies that the length of storage does not significant influence the $\mathrm{pH}$ of the sampled sachet water, which is similar to the report of Isikwue and Chikezie (2014). Similar to the first and third week, Mugulbu Water exhibited 
the highest $\mathrm{pH}$ value while AdsuMubihad the lowest $\mathrm{pH}$. Also, the electrical conductivity of the sachet water ranges from $0.02 \mathrm{~ms} / \mathrm{cm}^{3}$ to $0.23 \mathrm{~ms} / \mathrm{cm}^{3}$ and exhibited a significant variation of $46.15 \%$ just like the first and third week (see table 4 ).

\begin{tabular}{|c|c|c|c|c|c|c|c|c|c|c|c|c|c|}
\hline & 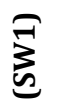 & 矛 & 管 & 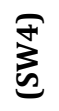 & $\underbrace{\frac{15}{3}}$ & 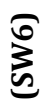 & 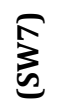 & 希 & 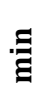 & ฐँ & ֻ & के & 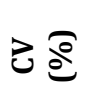 \\
\hline & 咅 & $\frac{\bar{v}}{\frac{3}{4}}$ & 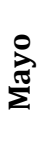 & 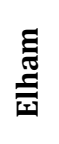 & $\sum_{3}^{\frac{\pi}{3}}$ & 莺 & $\frac{\pi}{z}$ & & & & & & \\
\hline colour & $\begin{array}{l}\frac{\tilde{u}}{0} \\
\frac{0}{0} \\
\frac{0}{0}\end{array}$ & $\begin{array}{l}\frac{\tilde{u}}{0} \\
\frac{0}{0} \\
\frac{0}{0}\end{array}$ & $\begin{array}{l}\frac{\mathscr{u}}{0} \\
\frac{0}{0} \\
\frac{0}{0}\end{array}$ & $\begin{array}{l}\frac{u}{0} \\
\frac{0}{0} \\
\frac{0}{0}\end{array}$ & $\begin{array}{l}\frac{\tilde{u}}{0} \\
\frac{0}{0} \\
\frac{0}{0}\end{array}$ & $\begin{array}{l}\frac{\mathscr{c}}{0} \\
\frac{0}{0} \\
\frac{0}{0}\end{array}$ & $\begin{array}{l}\frac{\tilde{u}}{0} \\
\frac{0}{0} \\
\frac{0}{0}\end{array}$ & ' & I & 1 & 1 & ' & 1 \\
\hline Odour & 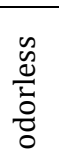 & 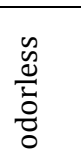 & 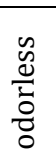 & $\begin{array}{l}\frac{w}{0} \\
\frac{0}{i} \\
\frac{0}{0}\end{array}$ & $\begin{array}{l}\frac{\tilde{u}}{0} \\
\frac{0}{\pi} \\
\frac{\pi}{0}\end{array}$ & $\frac{\mathscr{e}}{\frac{0}{0}}$ & 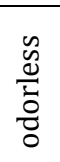 & I & I & ' & 1 & 1 & 1 \\
\hline $\begin{array}{l}\text { Temperature } \\
\text { (0c) }\end{array}$ & $\stackrel{+}{\stackrel{\sim}{\sim}}$ & 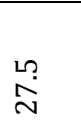 & 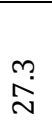 & $\stackrel{+}{\stackrel{N}{N}}$ & 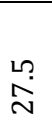 & $\stackrel{⿱ 亠}{N}$ & $\stackrel{0}{\stackrel{0}{\sim}}$ & $\begin{array}{l}\stackrel{8}{0} \\
\stackrel{N}{N}\end{array}$ & $\stackrel{\substack{n \\
\sim}}{\sim}$ & $\underset{\stackrel{+}{\sim}}{\stackrel{\sim}{N}}$ & $\stackrel{m}{0}$ & $\stackrel{\circ}{0}$ & $\stackrel{\substack{m \\
0}}{0}$ \\
\hline $\mathrm{pH}$ & $\underset{\infty}{\stackrel{D}{N}}$ & $\stackrel{ }{\stackrel{2}{r}}$ & $\stackrel{?}{\stackrel{2}{r}}$ & $\stackrel{\circ}{\stackrel{2}{n}}$ & $\stackrel{\curvearrowright}{N}$ & $\stackrel{\infty}{\infty}$ & $\stackrel{\circ}{\stackrel{2}{r}}$ & $\underset{\infty}{\stackrel{\infty}{N}}$ & $\stackrel{ }{7}$ & $\stackrel{8}{\circ}$ & $\stackrel{?}{\stackrel{ }{\rightarrow}}$ & 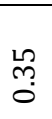 & $\stackrel{\vec{b}}{+}$ \\
\hline $\begin{array}{c}\text { Electrical } \\
\text { conductivity }\end{array}$ & $\begin{array}{l}\text { No } \\
\text { Oे }\end{array}$ & 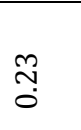 & $\stackrel{+}{\circ}$ & $\stackrel{n}{2}$ & $\stackrel{2}{2}$ & $\begin{array}{l}\infty \\
\stackrel{0}{0}\end{array}$ & ㄱ. & 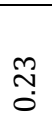 & $\begin{array}{l}\text { Tे } \\
\text { : }\end{array}$ & 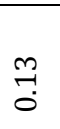 & $\stackrel{\text { స̆ }}{0}$ & $\stackrel{0}{0}$ & 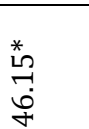 \\
\hline Turbidity & $\stackrel{\check{N}}{0}$ & $\underset{N}{N}$ & $\stackrel{N}{0}$ & 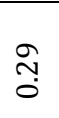 & m. & กุ? & $\ddot{n}$ & กิด & 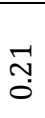 & $\ddot{m}$ & $\stackrel{\mathscr{m}}{0}$ & $\stackrel{\text { సै }}{0}$ & 离 \\
\hline $\begin{array}{c}\text { Total } \\
\text { Hardness } \\
\text { (mg/l }\end{array}$ & 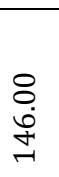 & $\begin{array}{l}8 \\
0 \\
\infty \\
\infty\end{array}$ & $\begin{array}{l}8 \\
\stackrel{8}{+} \\
-1\end{array}$ & $\underset{\text { İ }}{\stackrel{8}{ }}$ & $\begin{array}{l}\stackrel{8}{+} \\
+ \\
\infty\end{array}$ & 怘 & $\begin{array}{l}8 \\
\stackrel{0}{0} \\
\dot{m} \\
\rightarrow\end{array}$ & 뭉 & $\begin{array}{l}\stackrel{8}{+} \\
+ \\
\infty\end{array}$ & $\begin{array}{l}\infty \\
\infty \\
\infty \\
\exists\end{array}$ & $\underset{\substack{\infty \\
\infty}}{\stackrel{\infty}{\infty}}$ & 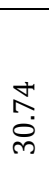 & 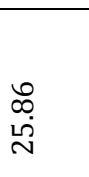 \\
\hline $\begin{array}{c}\text { Total } \\
\text { Dissolved } \\
\text { Solid (mg/l) }\end{array}$ & $\begin{array}{l}\dot{0} \\
\dot{m}\end{array}$ & 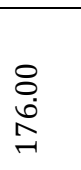 & $\begin{array}{l}8 \\
\circ \\
\circ \\
\circ\end{array}$ & $\begin{array}{l}\stackrel{8}{a} \\
\underset{\exists}{ت}\end{array}$ & $\begin{array}{l}\stackrel{8}{+} \\
\stackrel{+}{-}\end{array}$ & $\begin{array}{l}\text { ㅇ. } \\
\text { ํํ }\end{array}$ & $\begin{array}{l}8 \\
\text { fं }\end{array}$ & \begin{tabular}{l}
8 \\
0 \\
\multirow{2}{7}{} \\
7
\end{tabular} & $\begin{array}{l}\stackrel{8}{0} \\
\dot{0}\end{array}$ & $\begin{array}{l}\infty \\
\infty \\
\sigma\end{array}$ & $\begin{array}{l}8 \\
8 \\
0 \\
\stackrel{+}{+1}\end{array}$ & 离 & 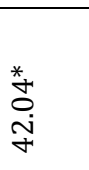 \\
\hline $\begin{array}{c}\text { Dissolved } \\
\text { Oxygen } \\
(\mathrm{mg} / \mathrm{l})\end{array}$ & $\begin{array}{l}8 \\
0 \\
0\end{array}$ & $\begin{array}{l}8 \\
0 \\
\infty \\
0\end{array}$ & $\begin{array}{l}8 \\
\text { Lी } \\
\text { Lी }\end{array}$ & $\begin{array}{l}8 \\
\text { iी }\end{array}$ & $\begin{array}{l}8 \\
0 \\
0\end{array}$ & $\stackrel{8}{i}$ & 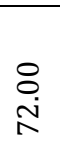 & 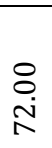 & $\begin{array}{l}8 \\
\text { : }\end{array}$ & $\stackrel{\sqrt{\hat{i}}}{\hat{6}}$ & $\stackrel{\circ}{\circ}$ & $\begin{array}{l}\text { กै } \\
0\end{array}$ & $\stackrel{N}{\underset{+}{*}}$ \\
\hline $\begin{array}{l}\text { Nitrate } \\
\text { (mg/l) }\end{array}$ & $\begin{array}{l}\stackrel{8}{0} \\
\stackrel{\infty}{N}\end{array}$ & $\begin{array}{l}\stackrel{8}{+} \\
\rightarrow \\
-1\end{array}$ & 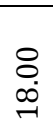 & 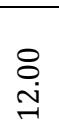 & 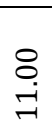 & $\begin{array}{l}\stackrel{8}{\circ} \\
\stackrel{+}{+}\end{array}$ & $\begin{array}{l}8 \\
\text { in } \\
\text { मे }\end{array}$ & $\begin{array}{l}\stackrel{8}{\infty} \\
\stackrel{\infty}{N}\end{array}$ & $\begin{array}{l}\stackrel{8}{\circ} \\
\stackrel{-}{-}\end{array}$ & 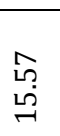 & 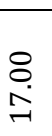 & $\begin{array}{l}\infty \\
\text { ho } \\
\text { h }\end{array}$ & 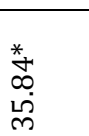 \\
\hline $\begin{array}{l}\text { Chlorine } \\
\text { (mg/l) }\end{array}$ & \& & $\begin{array}{l}\stackrel{8}{8} \\
\text { N }\end{array}$ & 8 & 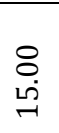 & $\begin{array}{l}8 \\
\substack{0 \\
\infty \\
-1}\end{array}$ & : & $\begin{array}{l}8 \\
\infty \\
\infty\end{array}$ & $\begin{array}{l}\stackrel{8}{\text { in }} \\
\text { N }\end{array}$ & $\begin{array}{l}8 \\
\text { ம் }\end{array}$ & $\underset{\sim}{\stackrel{+}{\leftrightarrows}}$ & $\begin{array}{l}\stackrel{8}{\circ} \\
\end{array}$ & $\begin{array}{l}\text { के } \\
\text { iे }\end{array}$ & 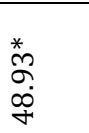 \\
\hline Iron (mg/) & $\stackrel{m}{0}$ & $\stackrel{m}{0}$ & $\overrightarrow{0}$ & 궁 & 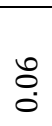 & : & $\begin{array}{l}1 \\
0 \\
0\end{array}$ & ?. & $\begin{array}{l}\text { mo } \\
0 \\
0\end{array}$ & Oे. & $\begin{array}{l}\infty \\
0 \\
0\end{array}$ & $\begin{array}{l}\text { On } \\
0 \\
0\end{array}$ & 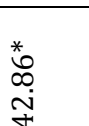 \\
\hline
\end{tabular}




\begin{tabular}{|c|c|c|c|c|c|c|c|c|c|c|c|c|c|}
\hline & 疍 & 旁 & 䇣 & 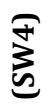 & 点 & $\sum^{0}$ & $\underbrace{5}$ & ્ֻ㐅 & 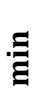 & 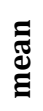 & ્ֻ0 & ڤิ & 己 \\
\hline & בె & 离 & 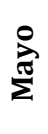 & 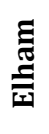 & 吾 & 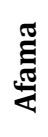 & z & & & & & & \\
\hline $\begin{array}{c}\text { Total } \\
\text { Bacterial } \\
\text { count } \\
(\mathrm{Cfu} / \mathrm{mol})\end{array}$ & 号 & $\begin{array}{l}\stackrel{0}{\overrightarrow{1}} \\
\stackrel{*}{\circ} \\
\stackrel{\text { iv }}{ }\end{array}$ & 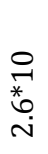 & $\begin{array}{l}\stackrel{0}{ } \\
\underset{*}{*} \\
\stackrel{N}{i} \\
\end{array}$ & $\begin{array}{l}\stackrel{0}{*} \\
\stackrel{F}{*} \\
\text { ñ }\end{array}$ & \begin{tabular}{l}
$\stackrel{\circ}{*}$ \\
\multirow{*}{*}{} \\
$\stackrel{N}{N}$
\end{tabular} & $\begin{array}{l}\stackrel{\circ}{*} \\
\stackrel{*}{*} \\
\stackrel{N}{i}\end{array}$ & $\begin{array}{l}\stackrel{0}{\vec{*}} \\
\stackrel{*}{n} \\
\dot{m}\end{array}$ & $\begin{array}{l}\stackrel{\circ}{*} \\
\stackrel{F}{*} \\
\stackrel{\text { N }}{1}\end{array}$ & $\begin{array}{l}\stackrel{0}{7} \\
\underset{*}{6} \\
\stackrel{i}{i}\end{array}$ & $\stackrel{\stackrel{\circ}{F}}{\stackrel{*}{\sim}}$ & లె & $\stackrel{\stackrel{P}{r}}{\stackrel{m}{\rightarrow}}$ \\
\hline $\begin{array}{c}\text { Total } \\
\text { Coliform } \\
\text { Count } \\
(\mathrm{Cfu} / \mathrm{mol})\end{array}$ & $\begin{array}{l}\stackrel{\circ}{F} \\
\stackrel{F}{n} \\
\stackrel{N}{i}\end{array}$ & $\begin{array}{l}\stackrel{\circ}{*} \\
\stackrel{F}{*} \\
\stackrel{i}{v}\end{array}$ & \begin{tabular}{l}
$\stackrel{0}{*}$ \\
\multirow{*}{*}{} \\
$\stackrel{-}{-}$
\end{tabular} & $\begin{array}{l}\stackrel{0}{2} \\
\vec{*} \\
\stackrel{0}{m} \\
\dot{m}\end{array}$ & $\begin{array}{l}\stackrel{0}{ } \\
\text { * } \\
\text { ì }\end{array}$ & $\begin{array}{l}\stackrel{ }{*} \\
\stackrel{*}{N} \\
\stackrel{i}{i}\end{array}$ & $\begin{array}{l}\stackrel{0}{7} \\
\text { * } \\
\dot{n}\end{array}$ & $\begin{array}{l}\stackrel{0}{7} \\
\underset{*}{*} \\
\stackrel{n}{n}\end{array}$ & $\begin{array}{l}\stackrel{0}{7} \\
\text { * } \\
\stackrel{-}{-}\end{array}$ & $\begin{array}{l}\stackrel{\circ}{*} \\
\stackrel{*}{*} \\
\stackrel{\text { i }}{ }\end{array}$ & $\begin{array}{l}\stackrel{\circ}{F} \\
\stackrel{*}{\wedge} \\
\stackrel{-}{-}\end{array}$ & นึ? & $\underset{+}{+}$ \\
\hline $\begin{array}{c}\text { E-Coli } \\
(\mathrm{Cfu} / \mathrm{mol})\end{array}$ & $\begin{array}{l}\circ \\
0 \\
0\end{array}$ & : & : & $\stackrel{8}{\circ}$ & $\begin{array}{l}\circ \\
0 \\
0\end{array}$ & $\begin{array}{l}8 \\
0 \\
0\end{array}$ & @: & $\stackrel{8}{\circ}$ & $\begin{array}{l}{ }_{0}^{\circ} \\
0\end{array}$ & $\begin{array}{l}8 \\
0\end{array}$ & $\begin{array}{l}\circ \\
\circ \\
0\end{array}$ & 1 & 1 \\
\hline
\end{tabular}

Table 4: Physiochemical and Biological Properties of Sachet Water in MubiMetropolis, Adamawa State (Week 5) $S D=$ Standard Deviation, $C V=$ Coefficient of Variation, Significant C.V $\geq 33 \%$

Source: Author's Compilation (2021)

Similar to the first week, the turbidity of the sampled sachet water ranges from $0.21 \mathrm{NTU}$ to $0.57 \mathrm{NTU}$, with a mean value of $0.31 \mathrm{NTU}$ and significant variation at 38.71\%, (see table 4). MayoWater exhibited the highest turbidity level of 0.57NTU while Mugulbu Water exhibited the lowest turbidity level of 0.21NTU. The total hardness of the samples increased slightly, ranging from $84 \mathrm{mg} / \mathrm{l}$ to $171 \mathrm{mg} / \mathrm{l}$, with a mean of $118.86 \mathrm{mg} / \mathrm{l}$ and an insignificant variation of $25.86 \%$ (see table 4). Similar to the first week, Elhamwas observed to have the highest total hardness level of $171 \mathrm{mg} / \mathrm{l}$ while Whora have the lowest at $84 \mathrm{mg} / \mathrm{l}$.

The Total Dissolved Solid (TDS) content increased slightly when compared to the first and third week and ranges between $30.00 \mathrm{mg} / \mathrm{l}$ and $176 \mathrm{mg} / \mathrm{l}$, with a mean and significant CV value of $99.86 \mathrm{mg} / \mathrm{l}$ and $42.04 \%$ respectively (see table 4). AdsuMubi and Mugulbu Water still exhibited the highest and lowest TDS content respectively. On the other hand, the dissolved oxygen content of the sampled sachet water remains relatively homogenous with a ranges from $65.00 \mathrm{mg} / \mathrm{l}$ to $72 \mathrm{mg} / \mathrm{l}$ (see table 4), with reduced mean of $67.71 \mathrm{mg} / \mathrm{l}$ when compared to the first and fifth week. Elham and Afama Water indicated the lowest content at $65.00 \mathrm{mg} / \mathrm{l}$ while MayoWater and NjikiWater indicated the highest value at $72.00 \mathrm{mg} / \mathrm{l}$.

During the fifth week the nitrate content of the sampled sachet water ranges from $11.00 \mathrm{mg} / \mathrm{l}$ to $28.00 \mathrm{mg} / \mathrm{l}$, with a mean value of $15.57 \mathrm{mg} / \mathrm{l}$ and a significant variation at $35.84 \%$ (see table 4 ). Mugulbu Water exhibited the highest nitrate content of $28.00 \mathrm{mg} / \mathrm{l}$ while AdsuMubi and Whora exhibited the lowest nitrate content of $11.00 \mathrm{mg} / \mathrm{l}$. The chlorine content of the sampled sachet water reduced slightly when compared to the first week and third week. The chlorine content reduced to a range between $5.00 \mathrm{mg} / \mathrm{l}$ and $22.00 \mathrm{mg} / \mathrm{l}$, with a reduced mean value of $12.14 \mathrm{mg} / \mathrm{l}$ and a significant variation at $48.93 \%$ indicating heterogenic chlorine content within the sachet water (see table 4 ). The iron content of the sampled sachet water ranges from $0.03 \mathrm{mg} / \mathrm{l}$ to $0.11 \mathrm{mg} / \mathrm{l}$ and exhibited a significant variation (see table 4). The iron content of ElhamandAfamaWaterare highest at $0.11 \mathrm{mg} / \mathrm{l}$ while Mugulbu Water and AdsuMubi exhibited the lowest iron content at $0.03 \mathrm{mg} / \mathrm{l}$. The Total Coliform Count (TBC) of the sampled sachet water increased when compared to the first and third week to a range between $2.0^{*} 10^{1}$ and $3.2^{*} 10^{1}$, with an increased mean of $2.6^{*} 10^{1}$. Similar to the result of the earlier weeks, Opeperfectal has the highest TBC content of $3.2^{*} 10^{1}$ while AsuMubi has the lowest of $2.0^{*} 10^{1}$. Also, the Total Coliform Count (TCC) increased slightly and ranges between $1.9^{*} 10^{1}$ and $3.6^{*} 10^{1}$, with a mean and insignificant variation of $2.7^{*} 10^{1}$ and $18.44 \%$ respectively. On the other hand, E.coli remained absent in all of the sampled sachet water (see table 4).

\section{Summary}

All of the sampled sachet water was colourless and odourless throughout the study interval, making them suitable for drinking as they fall within the WHO (2020) and NIS/NSDWQ (2020) requirements of $\leq 5$ and $\leq 15$ requirements. The temperature of the sachet water ranges from $27.3^{\circ} \mathrm{Cto} 27.6^{\circ} \mathrm{C}$ during the period of study. The temperature of the sachet water falls below the $40^{\circ} \mathrm{C}$ maximum permissible level prescribed by WHO (2020). The pH of all the sampled sachet water ranges between 7.10 and 8.20, which falls within the 6.6-8.5 prescribed by WHO (2020), NIS/NSDWQ (2020) and SON (2020). It was also observed that the length of storage does not influence sampled sachet water pH. The EC level of all the sachet water ranges between $0.02 \mathrm{~ms} / \mathrm{cm} 3$ and $0.15 \mathrm{~ms} / \mathrm{cm} 3$, which falls within the $\leq 1 \mathrm{~ms} / \mathrm{cm} 3 \mathrm{required}$ by WHO (2020), NIS/NSDWQ (2020) and SON (2020).

The turbidity level of all the sachet water ranges between $0.21 \mathrm{NTU}$ and $0.57 \mathrm{NTU}$, which falls within the $\leq 5 \mathrm{NTU}$ recommended by WHO (2020) and NIS/NSDWQ (2020). Some of these levels exceeds the level recommended by WHO 
(2020), NIS/NSDWQ (2020) and SON (2020). Mugulbu water, Mayo water and Njikiwater exceeded the $\leq 100 \mathrm{mg} / \mathrm{l}$ recommended by WHO (2020) and SON (2020) throughout the study period while Elhamexceeded the $\leq 150 \mathrm{mg} / \mathrm{l}$ recommended by NIS/NSDWQ and $\leq 100 \mathrm{mg} / \mathrm{l}$ recommended by WHO (2020) and SON (2020) throughout the study period. The level of TDS in all the sachet water ranges between $26 \mathrm{mg} / \mathrm{l}$ and $176 \mathrm{mg} / \mathrm{l}$, which falls within the $\leq 500 \mathrm{mg} / \mathrm{l}$ recommended by WHO (2020), NIS/NSDWQ (2020) and SON (2020). The dissolved oxygen level of the sachet water ranges from $65 \mathrm{mg} / \mathrm{l}$ and $78 \mathrm{mg} / \mathrm{l}$. The nitrate level in the sachet water ranges between $10 \mathrm{mg} / \mathrm{l}$ and $28 \mathrm{mg} / \mathrm{l}$, which all falls within the $\leq 50 \mathrm{mg} / \mathrm{l}$ level recommended by WHO (2020) and NIS/NSDWQ (2020). However, some of these levels exceeded the $\leq 10 \mathrm{mg} / \mathrm{l}$ level recommended by SON (2020). Specifically, Mugulbu water, Elhamwater, Afamawater, Mayowater and Njiki water all exceeded the $\leq 10 \mathrm{mg} / \mathrm{l}$ level recommended by SON $(2020)$. Notably, AdsuMubi $(11 \mathrm{mg} / \mathrm{l})$ and Whora $(11 \mathrm{mg} / \mathrm{l})$ slightly exceeded the $\leq 10 \mathrm{mg} / \mathrm{l}$ level recommended by SON (2020) on the fifth week of the study. The chlorine level of the sampled sachet water ranges between $5 \mathrm{mg} / \mathrm{l}$ and $26 \mathrm{mg} / \mathrm{l}$, which falls within the $\leq 250 \mathrm{mg} / \mathrm{l}$ recommended by WHO (2020) and NIS/NSDWQ (2020).

Although, chlorine has no adverse health impact, excess of it impacts bad taste to the drinking water. The level of iron in the sampled sachet water ranges between $0.02 \mathrm{mg} / \mathrm{l}$ and $0.11 \mathrm{mg} / \mathrm{l}$, which falls within the $\leq .03 \mathrm{mg} / \mathrm{l}$ recommended by WHO (2020), NIS/NSDWQ (2020) and SON (2020). The level of TBC in all the sachets water ranges between $1.8^{*} 101 \mathrm{Cfu} / \mathrm{mol}$ and $3.1^{*} 101 \mathrm{Cfu} / \mathrm{mol}$. These levels of TBC are unsuitable for drinking, as the level of TBC in all of the sampled sachet water exceeded the WHO (2020), NIS/NSDWQ (2020) and SON (2020) recommended level of 0Cfu/mol. This finding corresponds with the findings of Martins and Ada (2014) who reported bacterial pollution in all sampled sachet water brands in Bauchi metropolis, Nigeria. Furthermore, the notable increase in the TBC content form between $1.8^{*} 101 \mathrm{cfu} / \mathrm{mol}$ and $3.1 * 101 \mathrm{cfu} / \mathrm{mol}$ in the first week to between $2.0 * 101 \mathrm{cfu} / \mathrm{mol}$ and $3.2 * 101 \mathrm{cfu} / \mathrm{mol}$ in fifth week is in line with the findings of Akinde, et al, (2011) that reported increase in bacteria count in sachet water in Port Harcourt when stored beyond four weeks.

The level of TCC in all the sachets water ranges between $1.6 * 101 \mathrm{Cfu} / \mathrm{mol}$ and $3.0^{*} 101 \mathrm{Cfu} / \mathrm{mol}$. These levels of TCC are unsuitable for drinking, as the level of TCC in all of the sampled sachet water exceeded the NIS/NSDWQ (2020) and SON (2020) recommended level of $1 * 101 \mathrm{Cfu} / \mathrm{mol}$. The total coliform values of the sampled sachet water were well above the NIS/NSDWQ (2020) and SON (2020) values indicating that they are contaminated. E.coli was absent in all of the sampled sachet water throughout the study period as recommended by WHO (2020), NIS/NSDWQ (2020) and SON (2020) that drinking water should not contain any level of faecal constituent (WHO, 1971). The absent of E.coli indicates that all the water samples are free from recent faecal contamination (WHO, 1996; Dada, 2009).The total hardness level in the sachet water ranges between $81 \mathrm{mg} / \mathrm{l}$ and $171 \mathrm{mg} / \mathrm{l}$.

\section{Conclusion}

This study assessed the physiochemical and biological properties of sachet water produced in Mubi metropolis, Adamawa state for a period of five weeks. The study was directed towards examining the nature, properties and variation that exist within seven brands of sachet water produced in the area. Samples of sachet water were acquired immediately after they were produced from their various companies and exposed to laboratory analysis for a period of five weeks to derive relevant information that influences water portability.The study revealed that the physiochemical and biological properties of the sampled sachet water remain fairly the same all through the study period.

The study reveals that most of the sachet water analyzed were satisfactory in terms of all of the physical characteristics, except hardness which has not been attributed to any ill health. In terms of the chemical characteristics, most of the sampled sachet water met the recommended standards, except for nitrate which falls within the WHO (2020) and NIS/NSDWQ (2020) standard of $\leq 50 \mathrm{mg} / \mathrm{l}$ but questionable when compared to SON (2020) standards of $10 \mathrm{mg} / \mathrm{l}$. However, due to the associated effect of excess nitrate in drinking water on infants, it is recommended that some of these sachets water should be avoided when it comes to infant's consumption.

The microbial characteristics of all of the sachet water sampled contained coliforms (TBC and TCC) above recommended standards, thus making them unsafe for drinking. It was discovered from the study that the major contaminant of sachet water produced in Mubi metropolis, Adamawa state are the coliforms (TBC and TCC). These can constitute serious health challenges of diarrhea, meningitis, urinary tract infection dysentery, typhoid fever, paratyphoid fever, cholera, infantile paralysis, poliomyelitis, infectious hepatitis, guinea worm, amoebic dysentery and hemolytic prevalent in the area.

\section{Recommendation}

NAFDAC should carry out regular and frequent surveys of sachet water quality analysis for proper monitoring and management of sachet water production and its quality, Sachet water producing companies should endeavor to carry out proper treatment of the water they produce to ensure it is free of pathogens. Due to the associated effect of excess nitrate in drinking water on infants, it is recommended that some of these sachet waters be avoided for infant's consumption. This study recommends further studies to investigate the life of sachet water in the area beyond 5 weeks and to critically examine the biological quality of the sachet water produced in the area. 


\section{Implication}

The implication of this finding is the possibility of the presence of pathogens that may cause acute intestinal illness, which generally cause discomfort to health and could become fatal for some susceptible groups such as infants, elderly and sick people (Olowe, et al, 2005; Addo et al, 2009; NSDWQ, 2007). Therefore, there is an urgent need for awareness to be created about the biological status of these sachets water to enlighten the sachet water producing companies and customers on the necessity for further treatment of these sachet water before they are consider fit for drinking and domestic purposes.

\section{References}

i. Adediji A, Ajibade LT (2005). Quality of Well Water in Ede Area, South West Nigeria.J. Hum. Ecol. 17(3):223-228

ii. Adetunji, B.M. and Ilias, B.M. (2010). Externality Effect of Sachet Water Consumption and the Choice of Policy Instruments in Nigeria: Evidence from Kwara State. Journal of Economics, 1(2):113-131.

iii. Adewale, B.Z. (2017). Assessment of Groundwater Potential for Rural Water Supply Scheme Using Geographic Information System (GIS) in Ede North LGA of Osun State Nigeria.

iv. Adline C and Alaso J. (2015) A Comparative Study on the Measurement of PH of Water, Using

v. PH Metreand Water Testing Kit [Testube Method] in Port Harcourt. https://Www.Researchgate.Net/Publication/283270114

vi. Akinde, S.B., Nwachukwu, M.I., and Ogamba, A.S. (2011). Storage Effects on the Quality of Sachet Water Produced within Port Harcourt Metropolis Nigeria. Journal of Biological Sciences, 4: 157-164.

vii. Akpen, G.D., Kpogho, I.S. and Oparaku, L.A. (2018). Quality Assessment of Sachet and Bottled Water Sold in Gboko, Benue State, Nigeria. Nigerian Journal of Technology, 37(1): 241

viii. Akpoborie, I.A. and Ehwarimo, A. (2012). Quality of Packaged Drinking Water Produced in Warri Metropolis and Potential Implications for Public Health. Journal of Environmental Chemistry and Ecotoxicology, 4(11): $195-202$.

ix. APHA (1998).Standard Methods for the Examination of Water and Wastewater, 20th Edition, American Public Health Association, Washington D. C.

x. APHA, (1985).Standard Methods for Examination of Water and Wastewater, 20th Edition, American Public Health Association, Washington D. C.

xi. ASTM International (2003). Annual Book of ASTM Standards, Water and Environmental Technology, West Conshohocken, Pennsylvania, 11(1) 6-7.

xii. Bruvold, W.H. and Ongerth, H.J. (1969). Taste Quality of Mineralized Water. J. Am. Water Works Assoc., 61-170.

xiii. Cairncross, S. (2002). Cairncross, S. (1987). Water Supply and Sanitation: an agenda for research. J. Trop.Med. Hyg. 92:301-314.

xiv. Dada, A.C. (2006). Sachet Water Phenomenon in Nigeria: Assessment of the Potential Health Impacts. African Journal of Microbiological Research, 3(1): 15-21.

xv. DeGrandpre, M.D. (1993). Measurement of Seawater Pco2 Using a Renewable-Reagent Fiber Optic Sensor with Colorimetric Detection. Analytical Chemistry, 65: 331-337.

xvi. Denloye, S.A. (2004). Quality Parameters for Packaged Water, NAFDAC Laboratory Experience.IPAN News.

xvii. Federal Ministry of Environment (2002).National Guidelines and Standard for Water. Lagos,

xviii. Kodarkar, M.S. (1992). Methodology for Water Analysis, Physico-Chemical, Biological and Microbiological Indian Association of Aquatic Biologists Hyderabad, Pub. 2: 50.

xix. Minnesota Pollution Control Agency (2008). Turbidity: Description, Impact on Water Quality,Sources,Measures.Water Quality/Impaired Waters \#3.21.

xx. Mohammad R. I., Mohammad K. S., Tanzina A., Shafkat S. R., Rabiul I. T., Barun H., Md. A.

xxi. K. (2016) AStudy on Total Dissolved Solids and Hardness Level of Drinking Mineral Water in Bangladesh.American Journal of Applied Chemistry. Vol. 4, No. 5, pp. 164-169. doi: 10.11648/j.ajac.20160405.11

xxii. Mustafa, A.I, Ibrahim, A.A., Haruna, Y.I. and Abubakar, S. (2013). Physicochemical and Bacteriological Analyses of Drinking Water from Wash Boreholes in Maiduguri Metropolis, Borno State, Nigeria.African Journal of Food Science, 7(1): 9-13.

xxiii. Nayla H. O. (2019) Water Quality Parameters Open access peer-reviewed chapter DOI:

xxiv. 10.5772/intechopen.89657.

xxv. Oladipo, I.C., Onyenike, I.C. and Adebiyi, A.O. (2009).Microbiological Analysis of Some Vended Sachet Water in Ogbomoso, Nigeria.African Journal of Food Science, 3(12): 406-412.

xxvi. Olowe, O.A., Ojurongbe, O., Opaleye, 0.0., Adedosu, O.T., Oluwe, R.A. and Eniola, K.I.T. (2005).Bacteriological Quality of Water Samples in OsogboMetropolis.Afr. J. Clin. Exper.Microbiol., 6(3): 219-222.

xxvii. Omalu, I.C.J., Eze, G.C., Olayemi, I.K., Gbesi, S., Adeniran, L.A., Ayanwale, A.V., Mohammed, A.Z. and Chukwuemeka, V. (2011). Contamination of Sachet Water in Nigeria: Assessment and Health Impact.

xxviii. Osun Fact File (2019) Retrieved from http://osun.gov.ng/2017/02/25/osun-fact-file-socio-economic-culturalbackground/ on 26/02/2019.

xxix. Oteze, G.E. (1991). Portability of Ground water of the Rima group of Aquifers in the SokotoBasin.Nigeria Journal of Geology and Mining, 27(1): 17-21.

xxx. Pankaj K. R., Nihar R. S., Mihir P. and Malabika B. R. (2015) Electrical Conductivity of Lake

xxxi. Water asEnvironmental Monitoring -A Case study of Rudrasagar Lake

https://www.researchgate.net/publication/275353467

xxxii. Patterson H. H., Johnson P. S., and W. B. (2003). Epperson Effect of Total Dissolved Solids and 
xxxiii. Sulfates inDrinking Water for Growing Steers Proceedings, Western Section, American Society of Animal Science Vol. 54.

xxxiv. Rahmanian N., Siti H. A., Homayoonfard M., Ali N. J., Rehan M., Sadef Y., and Nizami A. S.

xxxv. (2015)Analysis of Physiochemical Parameters to Evaluate the Drinking Water Quality in the State of Perak, Malaysia.

xxxvi. Standard Organisation of Nigeria (2007).Nigerian Standards for Drinking Water Quality. Nigerian Industrial Std, NIS 554, Published by the Standard Organisation. of Nigeria, 13/14 V.A. Street off Admiralty way, Lekkis, Lagos Nigeria.

xxxvii. Trivedy, R.K. and Goel, P.K., (1986). Chemical and Biological Methods for Water Pollution Studies, Environmental Publication, Karad, Maharashtra.

xxxviii. Wang, Z., Wang, Y., Cai, W.J. and Liu, S. Y, (2002). A Long Lathlength Spectrophotometric Pco Sensor Using a GasPermeable Liquid-Core Waveguide.Talanta, 57: 69-80.

xxxix. WHO (1996).Total Dissolve Solid in Drinking Water.Guideline for Drinking Water Quality.

xl. World Health Organization (2020).PH in Drinking-water.Background document fordevelopment ofWHOGuidelines for Drinking-water Quality.

xli. Yonnana, E., Adebayo, A.A. \& Mahmud, H. (2008). Assessment of channel banks stability inthe middle course of river Yedzeram, Mubi, Adamawa State. Nigerian Journal of Research and Production.Enugu; Nigerian Researchers Forum. 13(2); 144-152.

xlii. Yonnana, E., Dzarma, M.S. \& Oliver, K. (2020). Assessment of Channel Planform Adjustmentsin the Mubi Section of River Yedzeram, Adamawa State, Northeastern Nigeria. Jalingo Journal of Social and Management Sciences, 2(3); 176-193. 